\title{
A diagnosis of mycosis fungoides in a pediatric patient with recurrent Langerhans cell histiocytosis
}

\section{To the Editor:}

An 8-year-old female presented with a reddish-brown papule that tripled in size. She had a history of intermittent hypopigmentation (Fig. 1A). Biopsy of the papule revealed a lymphohistiocytic infiltrate with increased CD1a and S100-positive Langerhans cells, consistent with Langerhans cell histiocytosis (LCH) (Fig. 1D). v-RAF murine sarcoma viral oncogene homolog (BRAF) mutation testing was negative. Workup for other sites of disease was negative.

There was minimal response to topical steroids. She began oral prednisone with partial response but flared with taper. Treatment with oral methotrexate and prednisone ${ }^{1}$ resulted in complete response. With discontinuation, she had recurrence of papules and hypopigmentation. Repeat biopsy confirmed LCH. Methotrexate was resumed and she had full resolution of papules but persistent hypopigmentation; however, her papules recurred with discontinuation of therapy.

Repeat biopsy revealed atypical epidermotropic CD8-positive $T$ cells that displayed loss of CD2 and diminished expression of CD7
(Fig. 1C). A T-cell receptor gene rearrangement study identified a clonal T-cell population. CD1a did not reveal increased Langerhans cells. No clonal T-cell population was identified in prior biopsies. The patient was diagnosed with hypopigmented mycosis fungoides (MF), stage IB. She started treatment with topical triamcinolone and narrowband ultraviolet $B$ with complete resolution of her hypopigmentation (Fig. 1B).

LCH describes an abnormal proliferation of usually clonal LCs and commonly occurs in children with varying clinical presentations. It is thought to be an inflammatory myeloid neoplasm as there is a high rate of recurrent mutations of the MAP2K pathway, and transcriptional profiling suggests that LCs are similar to bone marrow derived monocyte and dendritic cell precursors. ${ }^{2}$ Additionally, $\mathrm{LCH}$ has been described to be associated with many other neoplasms ${ }^{3}$ and may precede, follow, or occur simultaneously. It is unclear in our patient which entity occurred first as she had no prior biopsies of the hypopigmentation.
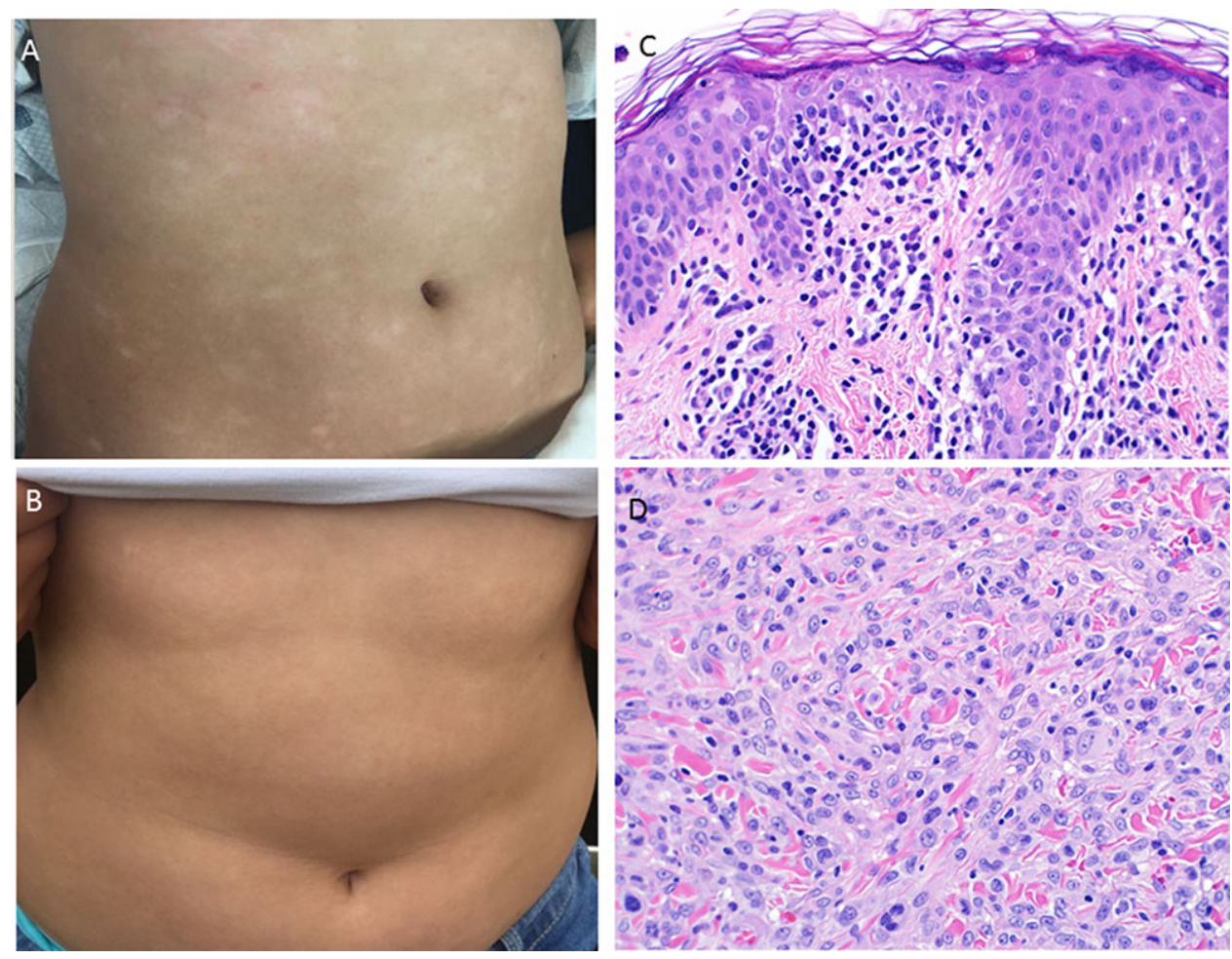

FIGURE 1 (A) Diffuse erythematous thin plaques with fine scale as well as hypopigmented macules on the anterior trunk, approximately 1 year after the initial diagnosis. (B) Significant repigmentation of the hypopigmented lesions approximately 8 months after starting phototherapy. (C) A biopsy of an erythematous papule in a background hypopigmented patch demonstrated cytologically atypical epidermotropic T cells (400X). (D) An excision of a large and rapidly growing papule revealed numerous scattered and collected Langerhans cells with reniform nuclei, longitudinal nuclear folds, and relatively abundant pale, eosinophilic cytoplasm (400x) 
Cutaneous T-cell lymphoma is a rare disease with an estimated incidence of 10.2 per million persons. ${ }^{4,5}$ It is uncommon in children, with peak incidence at 80 years. ${ }^{6} \mathrm{~A}$ review of the international Childhood Registry for Cutaneous Lymphomas revealed increased incidence in males and median age of diagnosis of 10 years. ${ }^{7}$ MF is the most common of the cutaneous T-cell lymphomas in all ages. ${ }^{8,9}$ It is typically presented as hypo- or hyperpigmented lesions, patches, plaques, or poikiloderma. Hypopigmented lesions predominate in younger patients.

A literature review highlights two cases of MF associated with $\mathrm{LCH} .{ }^{10}$ Both were older adult males diagnosed with MF with CD1a and S100 positive LCs admixed within biopsy samples. These differ from our case in that the nodules of LCs occurred concurrently, as is common in cases of LCH-associated lymphoma. Interestingly, our patient demonstrated LCH on early biopsies but all recent biopsies showed MF without LCH. To our knowledge, this is the first pediatric case of a patient with initial diagnosis of $\mathrm{LCH}$, responsive to treatment with multiple recurrences, and subsequent diagnosis of MF. This case should prompt clinicians caring for patients with LCH to be vigilant for the presence or development of hypopigmented patches/plaques that could represent MF and require a different treatment strategy.

\section{ETHICS STATEMENT}

Informed consent (from the patient's mother) was obtained and properly documented.

\section{CONFLICT OF INTEREST}

The authors declare that there is no conflict of interest.

$$
\begin{array}{r}
\text { Angela C. Weyand }^{1} \text { iD } \\
\text { Alexandra Hristov } \\
\text { Trilokraj Tejasvi }^{3} \\
\text { Kelly Walkovich }^{1}
\end{array}
$$

${ }^{1}$ Department of Pediatrics, University of Michigan, Ann Arbor, Michigan

${ }^{2}$ Department of Pathology, University of Michigan, Ann Arbor, Michigan

${ }^{3}$ Department of Dermatology, University of Michigan, Ann Arbor, Michigan
Correspondence

Angela C. Weyand, Department of Pediatrics, University of Michigan, MSRB III Room 8346, 1150 W. Medical Center Drive, Ann Arbor, MI 48109. Email:acweyand@med.umich.edu

\section{REFERENCES}

1. Womer RB, Anunciato KR, Chehrenama M. Oral methotrexate and alternate-day prednisone for low-risk Langerhans cell histiocytosis. Med Pediatr Oncol. 1995;25(2):70-73.

2. Haroche J, Cohen-Aubart F, Rollins BJ, et al. Histiocytoses: emerging neoplasia behind inflammation. Lancet Oncol. 2017;18(2):e113e125.

3. Egeler RM, Neglia JP, Arico M, et al. The relation of Langerhans cell histiocytosis to acute leukemia, lymphomas, and other solid tumors. The LCH-Malignancy Study Group of the Histiocyte Society. Hematol Oncol Clin North Am. 1998;12(2):369-378.

4. Korgavkar K, Xiong $\mathrm{M}$, Weinstock $\mathrm{M}$. Changing incidence trends of cutaneous T-cell lymphoma. JAMA Dermatol. 2013;149(11):12951299.

5. Billings SD, Hans CP, Schapiro BL, et al. Langerhans cell histiocytosis associated with myelodysplastic syndrome in adults. J Cutan Pathol. 2006;33(2):171-174.

6. Bradford PT, Devesa SS, Anderson WF, Toro JR. Cutaneous lymphoma incidence patterns in the United States: a population-based study of 3884 cases. Blood. 2009;113(21):5064-5073.

7. Pope E, Weitzman S, Ngan B, et al. Mycosis fungoides in the pediatric population: report from an international Childhood Registry of Cutaneous Lymphoma. J Cutan Med Surg. 2010;14(1):16.

8. Wain EM, Orchard GE, Whittaker SJ, Spittle MSMF, RussellJones R. Outcome in 34 patients with juvenile-onset mycosis fungoides: a clinical, immunophenotypic, and molecular study. Cancer. 2003;98(10):2282-2290.

9. Castano E, Glick S, Wolgast L, et al. Hypopigmented mycosis fungoides in childhood and adolescence: a long-term retrospective study. J Cutan Pathol. 2013;40(11):924-934.

10. Christie LJ, Evans AT, Bray SE, et al. Lesions resembling Langerhans cell histiocytosis in association with other lymphoproliferative disorders: a reactive or neoplastic phenomenon? Hum Pathol. 2006;37(1):3239. 